\title{
poinçonnement d'un sol élastique anisotrope
}

par

\author{
M. Dahan \\ Ingénieur, Attaché de Recherches C.N.R.S. \\ Laboratoire de Mécanique des solides*
}

\section{Introduction}

Pour la prévision des tassements, il est courant en Mécanique des Sols de simplifier le comportement des terres et de les considérer comme des corps élastiques pour obtenir des résultats approchés.

Jusqu'à présent, la plupart des calculs ont été faits en utilisant comme modèle mathématique le massif semi-infini. homogène, élastique et isotrope. Des solutions à ce problème ont été données par Boussinesq dès 1885. En fait, le sol n'est pas parfaitement isotrope, et cela peut expliquer certains désaccords entre la théorie et l'expérience.

Le simple dépôt, la sédimentation, la consolidation du sol sous l'effet du poids propre, peuvent entrainer l'apparition d'une anisotropie. Les propriétés du sol dans les directions horizontales et verticales ont alors des différences significatives. En supposant la symétrie des propriétés élastiques du sol autour de la verticale, on peut le considérer comme un massif semi-infini transversalement isotrope. Nous avons vérifié cette propriété sur divers échantillons de sols par la mesure des coefficients élastiques.

Le but de cet étude est de donner la distribution des contraintes et des déplacements à l'intérieur d'un sol à isotropie transversale lorsqu il est soumis à des chargements axisymétriques sur sa surface libre, puis de déterminer la répartition des pressions sous un poinçon rigide, ainsi qu'une relation entre la force de poinçonnement et la profondeur de pénétration du poinçon dans le sol.

Les résultats obtenus montrent que la distribution des pressions sous le poinçon est la même que si le massif était isotrope ; pour le tassement à la surface du massif, il suffit simplement de remplacer le module $2\left(1-v^{2}\right) / E$ du cas isotrope par un certain coefficient q. Nous généralisons ainsi tous les résultats connus pour le massif isotrope, à partir de la formule de Boussinesq, en ce qui concerne la distribution des contraintes sous le poincon et le tassement en surface, au milieu transversalement isotrope. Ces résultats sont illustrés par un certain nombre de courbes montrant l'influence de l'anisotropie sur les contraintes et les déplacements.

- Le Laboratoire de Mécanique des Solides est une équipe de Recherche associée au C.N.R.S. (Groupe Commun Ecole Polytechnique, Ecole Nationale Supérieure des Mines de Paris, Ecole Nationale des Ponts et Chaussées).

\section{Chargements axisymétriques.}

Nous nous proposons de généraliser au cas d'un chargement quelconque la méthode que nous avons utilisée (Dahan, 1975; Dahan et Zarka, 1977) pour la résolution du contact élastique d'une sphère avec un massif semi-infini.

Considérons un massif semi-infini transversalement isotrope dont la surface est délimitée par le plan horizontal ( $r$. $\theta$ ) et dont l'axe de symétrie élastique $(z)$ est vertical. Ce matériau est défini par cinq coefficients élastiques $\mathrm{a}_{\mathrm{ij}}$.

Les relations contraintes-déformations associées aux problèmes à symétrie axiale sont :

$$
\begin{aligned}
& \varepsilon_{r r}=a_{11} \sigma_{r r}+a_{12} \sigma_{\theta \theta}+a_{13} \sigma_{z z^{\prime}} \\
& \varepsilon_{\theta \theta}=a_{12} \sigma_{r r}+a_{11} \sigma_{\theta \theta}+a_{13} \sigma_{z z^{\prime}} \\
& \varepsilon_{z z}=a_{13} \sigma_{r r}+a_{13} \sigma_{\theta \theta}+a_{33} \sigma_{z z^{\prime}} \\
& \gamma_{r z}=a_{44} \sigma_{r z^{\prime}}
\end{aligned}
$$

où les déformations sont définies à partir des composantes $\left(u_{r}, u_{\theta}=0, w\right)$ du déplacement par les relations :

$$
\begin{aligned}
& \varepsilon_{r r}=\frac{\partial u_{r}}{\partial r} \\
& \varepsilon_{\theta \theta}=\frac{u_{r}}{r} \\
& \varepsilon_{z z}=\frac{\partial w}{\partial z} \\
& \gamma_{r z}=\frac{\partial u_{r}}{\partial z}+\frac{\partial w}{\partial r}
\end{aligned}
$$

Les équations d'équilibre sont :

$$
\frac{\partial \sigma_{r r}}{\partial r}+\frac{\partial \sigma_{r z}}{\partial z}+\frac{\sigma_{r r}-\sigma_{\theta \theta}}{r}=0
$$

$$
\frac{\partial \sigma_{r z}}{\partial r}+\frac{\partial \sigma_{z z}}{\partial z}+\frac{\sigma_{r z}}{r}=0
$$


et les équations de compatibilité transformées par les relations (1) s'écrivent :

$$
\begin{aligned}
& \left(a_{11}-a_{12}\right)\left(\sigma_{r r}-\sigma_{\theta \theta}\right)-r \frac{\partial}{\partial r}\left(a_{12} \sigma_{r r}+a_{11} \sigma_{\theta \theta}+a_{13} \sigma_{z 2}\right) \\
& =0,
\end{aligned}
$$

$$
\begin{aligned}
& \frac{\partial^{2}}{\partial z^{2}}\left(a_{11} \sigma_{r r}+a_{12} \sigma_{\theta \theta}+a_{13} \sigma_{z z}\right) \\
& \quad+\frac{\partial^{2}}{\partial r^{2}}\left(a_{13} \sigma_{r r}+a_{13} \sigma_{\theta \theta}+a_{33} \sigma_{z z}\right)-a_{44} \frac{\partial^{2} \sigma_{r z}}{\partial r \partial z}=0 .
\end{aligned}
$$

Les conditions aux limites sont telles que :

a) pour $r$ ou $z$ infini :

$$
\begin{aligned}
& \sigma_{r r}=\sigma_{\theta \theta}=\sigma_{z z}=\sigma_{r z}=0, \\
& u_{r}=u_{\theta}=w=0,
\end{aligned}
$$

b) à la surface du massif $(z=0)$ :

$$
\begin{aligned}
& \sigma_{r z}(r, 0)=0 \\
& \sigma_{z z}(r, 0)=-p(r)
\end{aligned}
$$

où $p(r)$ désigne la distribution des pressions appliquée normalement à la surface du massif, fonction nulle à l'extérieur du contact.

Nous avons ainsi posé de (3) à (7) les équations mathématiques définissant le problème de mécanique considéré.

Introduisons une fonction intermédiaire $\varphi$ telle que :

$$
\begin{aligned}
& \sigma_{r r}=-\frac{\partial}{\partial z}\left(\frac{\partial^{2} \varphi}{\partial r^{2}}+\frac{b}{r} \frac{\partial \varphi}{\partial r}+a \frac{\partial^{2} \varphi}{\partial z^{2}}\right) \\
& \sigma_{\theta \theta}=-\frac{\partial}{\partial z}\left(b \frac{\partial^{2} \varphi}{\partial r^{2}}+\frac{1}{r} \frac{\partial r}{\partial \varphi}+a \frac{\partial^{2} \varphi}{\partial z^{2}}\right) \\
& \cdot \sigma_{z z}=\frac{\partial}{\partial z}\left(c \frac{\partial^{2} \varphi}{\partial r^{2}}+\frac{c}{r} \frac{\partial \varphi}{\partial r}+d \frac{\partial^{2} \varphi}{\partial z^{2}}\right) \\
& \sigma_{r z}=\frac{\partial}{\partial r}\left(\frac{\partial^{2} \varphi}{\partial r^{2}}+\frac{1}{r} \frac{\partial \varphi}{\partial r}+a \frac{\partial^{2} \varphi}{\partial z^{2}}\right)
\end{aligned}
$$

où les coefficients a, b, c, d sont définis comme suit:

$$
\begin{aligned}
& a=a_{13}\left(a_{11}-a_{12}\right) /\left(a_{11} a_{33}-a_{13}\right), \\
& b=\left[a_{13}\left(a_{13}+a_{44}\right)-a_{12} a_{33}\right] /\left(a_{11} a_{33}-a_{13}\right),
\end{aligned}
$$

$$
c=\left|a_{13}\left(a_{11}-a_{12}\right)+a_{11} a_{44}\right| /\left(a_{11} a_{33}-a_{13}\right) \text {, }
$$$$
d=\left(a_{11}-a_{12}^{2}\right) /\left(a_{11} a_{33}-a_{13}\right) \text {. }
$$

Nous avons montré (Dahan, 1975 ; Dahan et Zarka, 1977) que le problème est résolu si cette fonction s'écrit sous la forme :

(10) $\varphi(r, z)=$

$$
\frac{1}{f\left(s_{1}-s_{2}\right)} \int_{0}^{\infty}\left(p_{2} e^{-s_{1} m z}-p_{1} e^{-s_{2} m z}\right) p H(m) J_{0}(m r) \frac{d m}{m^{2}}
$$

avec les notations suivantes:

$$
\frac{s_{1}}{s_{2}}=\left\{\left(a+c \pm \sqrt{\left.(a+c)^{2}-4 d\right)} / 2 d\right]^{1 / 2},\right.
$$

$$
\begin{aligned}
& \mathrm{p}_{\mathrm{i}}=1-\mathrm{as}_{\mathrm{i}}^{2}, \\
& \mathrm{q}_{\mathrm{i}}=\mathrm{s}_{\mathrm{i}}^{2}\left(\mathrm{a}_{33} \mathrm{~d}-2 \mathrm{a}_{13} \mathrm{a}\right)-\mathrm{a}_{44}, \quad i=1,2 \\
& \mathrm{f}=(\mathrm{d}-\mathrm{ac}) / \sqrt{\mathrm{d} .} \\
& \lambda=(1-\mathrm{b}) / f, \\
& \mathrm{q}=\lambda\left(\mathrm{a}_{11}-\mathrm{a}_{12}\right)\left(\mathrm{s}_{1}+\mathrm{s}_{2}\right)
\end{aligned}
$$

et où $\mathrm{p}^{\mathrm{H}}(\mathrm{m})$ est la transformée de Hankel d'ordre zéro du chargement $p(r)$ :

$$
p^{H}(m)=\int_{0}^{\infty} r J_{0}(m r) p(r) d r .
$$

$J_{0}$ désigne la fonction de Bessel de première espèce et d'ordre zéro.

En introduisant l'expression (10) de la fonction intermédiaire dans les relations (8), nous obtenons pour les contraintes et les déplacements les expressions suivantes valables en tout point du massif semi-infini :

$$
\begin{aligned}
& \sigma_{r r}=\left[\lambda\left(s_{1} p_{2} D_{1}-s_{2} p_{1} D_{2}\right)-\left(s_{1} A_{1}-s_{2} A_{2}\right) / \sqrt{d}\right] /\left(s_{1}-s_{2}\right) \\
& \sigma_{\theta \theta}=\left[-\lambda\left(s_{1} p_{2} D_{1}-s_{2} p_{1} D_{2}\right)-\right. \\
& \left.\left[s_{1} p_{2}\left(b-a s_{1}^{2}\right) A_{1}-s_{2} p_{1}\left(b-a s_{2}^{2}\right) A_{2}\right] / f\right] /\left(s_{1}-s_{2}\right) \\
& \sigma_{z z}=\left(s_{2} A_{1}-s_{1} A_{2}\right) /\left(s_{1}-s_{2}\right) \\
& \sigma_{r z}=\left(C_{1}-C_{2}\right) /\left(s_{1}-s_{2}\right) \sqrt{d} \\
& u_{r}=-\lambda_{r}\left(s_{1} p_{2} D_{1}-s_{2} p_{1} D_{2}\right)\left(a_{11}-a_{12}\right) /\left(s_{1}-s_{2}\right) \\
& w=r\left(q_{1} p_{2} B_{1}-q_{2} p_{1} B_{2}\right) / f\left(s_{1}-s_{2}\right)
\end{aligned}
$$

où nous posons :

$$
\begin{aligned}
& A_{i}=\int_{0}^{\infty} e^{-m s_{i} z} J_{0}(m r) p^{H}(m) m d m, \\
& B_{i}=\frac{1}{r} \int_{0}^{\infty} e^{-m s_{i} z} J_{0}(m r) p^{H}(m) d m,
\end{aligned}
$$

$$
\begin{aligned}
& C_{i}=\int_{0}^{\infty} e^{-m s_{i} z} J_{1}(m r) p^{H}(m) m d m . \\
& D_{i}=\frac{1}{r} \int_{0}^{\infty} e^{-m s_{i} z} J_{1}(m r) p^{H}(m) d m .
\end{aligned}
$$

Nous avons ainsi déterminé pour tout chargement $p(r)$ le comportement du sol correspondant.

\section{Poinçons rigides axisymétriques}

Dans le cas du contact du sol avec un poinçon rigide, la fonction $\mathrm{p}$ introduite en (7) est à déterminer; nous la savons simplement nulle à l'extérieur du cercle de contact $\left(r>r_{0}\right)$. Les seules données du problème sont la forme du poinçon $f(r)$ et la profondeur de pénétration $w_{0}$ ou la force de poinçonnement $\mathrm{P}$. A l'intérieur du contact, au lieu de I'hypothèse (7), nous avons une condition aux limites en déplacement :

(15) $w(r, 0)=w_{0}-f(r) \quad r \leqslant r_{0}$. 
A la surface du massif, les conditions aux limites s'écrivent, d'après les relations (13) avec $z=0$, de la façon suivante :

$w(r, 0)=q \int_{0}^{\infty} J_{0}(m r) p^{H}(m) d m=w_{0}-f(r)$ si $0 \leqslant r \leqslant r_{0}$ (16)

$\sigma_{z z}(r, 0)=-p(r)=-\int_{0}^{\infty} m J_{0}(m r) p^{H}(m) d m=0$ si $r>r_{0}$

En supposant $p^{H}$ de la forme :

$$
p^{H}(m)=\int_{0}^{r}{ }^{r} \chi(t) \cos (m t) d t
$$

la condition (16) pour $r>r_{0}$ est identiquement vérifiée. Pour $r \leqslant r_{0}$, nous déduisons de cette même condition l'équation intégrale d'Abel:

$$
q \int_{0}^{r} \chi(t)\left(r^{2}-t^{2}\right)^{-1 / 2} d t=w_{0}-f(r)
$$

dont la solution s'écrit :

$$
\chi(t)=\frac{2}{q \pi}\left[w_{0}-t \int_{0}^{t} f^{\prime}(r)\left(t^{2}-r^{2}\right)^{-1 / 2} d r\right] .
$$

Pour des profils de poinçons réguliers, sans discontinuité de plans tangents, $\mathrm{p}(\mathrm{r})$ reste fini sur le bord du cercle de contact. Nous montrons (Dahan, 1979) que cette condition est équivalente à :

(20) $\chi\left(r_{0}\right)=0$.

De l'inégalité (19), nous déduisons pour $t=r_{0}$, l'importante relation entre la profondeur $w_{0}$ de pénétration du poinçon et le rayon $r_{0}$ du cercle de contact:
(21) $w_{0}=r_{0} \int_{0}^{b} f^{\prime}(r)\left(r_{0}^{2}-r^{2}\right)^{-1 / 2} d r$.

Nous avons aussi la répartition des pressions sous le poinçon et sa transformée en fonction de $\chi$ :

$$
p(r)=-\int_{r}^{r_{0}} \chi^{\prime}(t) t\left(^{2}-r^{2}\right)^{-1 / 2} d t \quad r \leqslant r_{0} .
$$

$$
p^{H}(m)=-\int_{0}^{6} \chi^{\prime}(t) \sin (m t) \frac{d t}{m} .
$$

Pour la force de poinçonnement, nous avons la relation

(23) $\mathrm{P}=2 \pi \int_{0}^{\mathrm{r}_{0}} \chi(\mathrm{t}) \mathrm{dt}$.

II est aussi facile d'exprimer le tassement à l'extérieur du contact :

(24) $w(r, 0)=q \int_{0}^{r_{0}} \chi(t)\left(r^{2}-t^{2}\right)^{-1 / 2} d t \quad r>r_{0}$.

En conclusion, les formules (19) et (22) permettent de déterminer la répartition des pressions $\mathrm{p}(\mathrm{r})$ sous le poinçon. L'expression (21) détermine le rayon du cercle de contact en fonction du déplacement imposé $w_{0}$ et du profil du poinçon. Nous avons ainsi ramené le problème du poincon rigide à celui d'un chargement axisymétrique par une distribution de pression $p(r)$ sur un cercle de rayon $r_{0}$; c'est le problème qui a été traité au paragraphe précédent.

Pour des poincons particuliers, le tableau suivant donne les principaux résultats (Dahan, 1979).

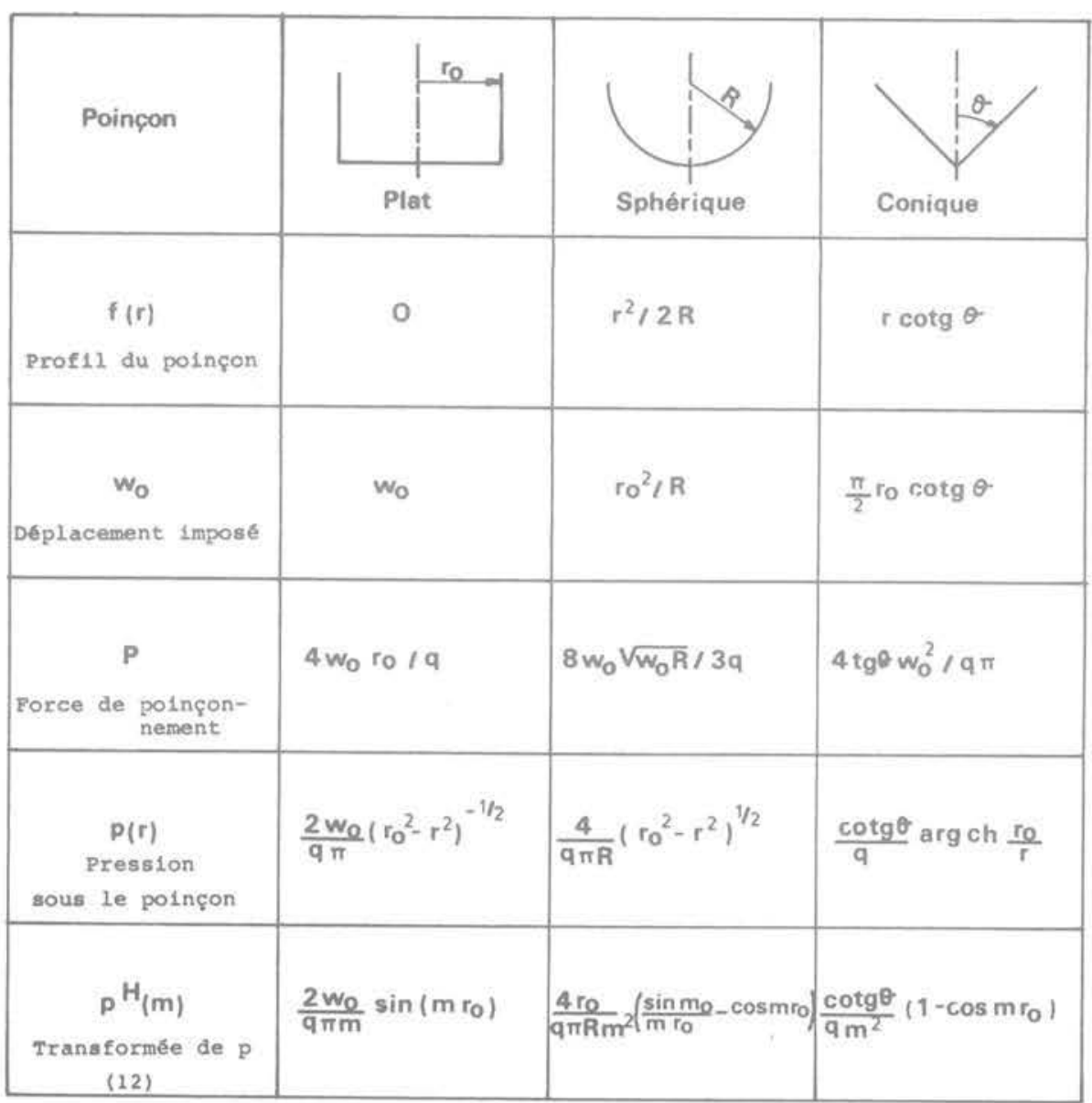




\section{Etude du coefficient $q$.}

D'après les formules (22) et (24), on remarque qu'à la surface du massif, la pression de poinçonnement $p(r)$ et les tassements $w$ peuvent être déduits de la solution isotrope (Sneddon, 1965) ; it suffit simplement de remplacer le coefficient q par le module $2\left(1-v^{2}\right) / E$ du cas isotrope.

Par conséquent, tous les résultats obtenus pour le massif isotrope à partir de la formule de Boussinesq en ce qui concerne la distribution des contraintes sous le poinçon et le tassement à l'intérieur comme à l'extérieur du contact sur la surface du massif, s'étendent au milieu à isotropie transversale.

Ces résultats apportent aussi une justification aux calculs pratiques de tassement de sols faits en utilisant un module expérimental obtenu à partir de l'enfoncement d'une plaque rigide sous une force donnée, comme le module de Westergaard (module de réaction). Ce module joue le rôle du coefficient q et tient compte de l'anisotropie.

Bien entendu, quand on s'écarte de la surface $z=0$, les résultats ne sont plus les mêmes que pour un massif isotrope. Pour montrer l'influence de l'anisotropie, nous donnons ci-dessous pour le poinçon plat et à titre de curiosité pour le poinçon conique, les isovaleurs de la contrainte normale $\sigma_{z z}$ et celles du déplacement vertical w pour deux sols anisotropes et deux sols isotropes tels que, dans chacun des deux cas, le coefficient q soit égal au module 2 $\left(1-v^{2}\right) / E$. Ces courbes ont été obtenues par un calcul numérique simple à partir des formules explicites (13) pour le cas anisotrope et des résultats de Sneddon (1946 et 1948). Les valeurs utilisées pour les coefficients $a_{i j}$ des sols anisotropes ont été mesurées au Laboratoire de Mécanique des Solides sur des éprouvettes de limon prélevé sur le plateau de Palaiseau et sur des éprouvettes d'une marne prélevée à Marseille. Ces deux sols présentent un sens d'anisotropie différent.

Des résultats présentés dans le tableau I et à partir des relations entre la force de poinçonnement et la profondeur de pénétration du poinçon, nous pouvons déduire un moyen expérimental très commode pour la mesure du coefficient $q$.

\section{Application pratique.}

Six éprouvettes de limon, de dimensions standard (hauteur : $72 \mathrm{~mm}$, diamètre : $36 \mathrm{~mm}$ ) ont été prélevées à Palaiseau à une profondeur d'environ $1,20 \mathrm{~m}$. Trois de ces éprouvettes ont été taillées dans le sens vertical, deux dans le sens horizontal et une à 45 degrés.

Sur ces éprouvettes, nous avons réalisé des essais de compression simple et avons obtenu les résultats suivants :

$$
\begin{aligned}
& E_{1}=3,910^{6} \mathrm{~Pa}, \\
& E_{2}=5,910^{6} \mathrm{~Pa}, \\
& v_{1}=0,10 \\
& v_{2}=0,13 \\
& \mu_{1}=\frac{E_{1}}{2\left(1+v_{1}\right)}=1,810^{6} \mathrm{~Pa}, \\
& \mu_{2}=2,410^{6} \mathrm{~Pa}, \\
& \text { d'où la valeur de } \mathrm{q}=3,610^{-7} \mathrm{~Pa}^{-1} .
\end{aligned}
$$

$E_{1}$ et $E_{2}$ sont les modules d'Young pour la direction parallèle aux plans d'isotropie du matériau et perpendiculaire à ceux-ci.

$v_{1}$ est le coefficient de Poisson qui caractérise la dilatation transversale dans le plan d'isotropie pour une compression parallèle à ce plan.

$v_{2}$ est le coefficient de Poisson qui caractérise la dilatation transversale dans le plan d'isotropie pour une compression de direction perpendiculaire à celui-ci. $\mu_{1}$ et $\mu_{2}$ sont les modules de cisaillement pour les plans d'isotropie et pour les plans perpendiculaires dans les directions radiales et on sait que $\mu_{1}$ n'est pas une valeur indépendante.

Nous rappeions que les coefficients élastiques $a_{i j}$ sont définis, pour un matériau transversalement isotrope, à partir de ces "constantes techniques " par les relations :

$$
\begin{aligned}
& a_{11}=\frac{1}{E_{1}}, \\
& a_{12}=-\frac{v_{1}}{E_{1}}, \\
& a_{13}=-\frac{v_{2}}{E_{2}}, \\
& a_{33}=\frac{1}{E_{2}} \\
& a_{44}=\frac{1}{\mu_{2}},
\end{aligned}
$$

Les résultats (25) montrent l'existence d'une certaine anisotropia, le rapport des modules d'Young $\frac{E_{1}}{E_{2}}$ est de l'ordre de 0,66 , c'est-â-dire que le limon parait plus raide dans le sens vertical que dans le sens horizontal.

La marne de Marseille a été prélevée à une cinquantaine de mètres de profondeur, et est en grande partie composée de montmorillonite et d'illite. Les essais en compression simple ont donné les résultats suivants:

$$
\begin{aligned}
& E_{1}=1,210^{8} \mathrm{~Pa}, \\
& E_{2}=3,910^{7} \mathrm{~Pa}, \\
& v_{1}=v_{2}=0, \\
& \mu_{1}=\frac{E_{1}}{2\left(1+v_{1}\right)}=610^{7} \mathrm{~Pa}, \\
& \mu_{2}=2,210^{7} \mathrm{~Pa},
\end{aligned}
$$

et ainsi : $\mathrm{q}=3,810^{-8} \mathrm{~Pa}^{-1}$.

Nous avons cette fois une anisotropie significativement différente, le rapport des modules $E_{1} / E_{2}$ est pratiquement égal à 3 , c'est-à-dire que la marne paraît plus raide dans le sens horizontal que dans le sens vertical.

Sur les courbes des figures 1 et 2 , nous remarquons que les isovaleurs de la contrainte normale relative au limon de Palaiseau sont au-dessus de celles obtenues pour le cas isotrope (trait discontinu), c'est-à-dire qu'en tout point du massif la contrainte $\sigma_{z z}$ anisotrope est supérieure à la contrainte $\sigma_{z z}$ isotrope, sauf sur la surface où elles sont égales. Nous avons le résultat contraire pour la marne de Marseille. Cette différence s'explique par le sens de l'anisotropie des deux sols puisque le rapport $E_{1} / E_{2}$ est inférieur à 1 pour le limon, alors qu'il est supérieur à 1 pour la marne.

Pour les tassements, on voit sur les figures 3 et 4 que les différences varient en fonction du rapport d'anisotropie. Nous avons ainsi de plus grands écarts avec le cas isotrope pour la marne que pour le limon.

\section{Conclusion}

Nous avons donné dans cette étude un calcul analytique permettant d'obtenir la distribution des contraintes et des déplacements en tout point d'un sol semi-infini transversalement isotrope soumis à des chargements axisymétriques sur sa surface libre. Cette méthode conduit pour la plupart des chargements classiques à des résultats explicites, dont l'utilisation est aisée.

Nous avons montré aussi que la répartition de la pression sous la fondation et le tassement en surface sont identiques à un coefficient multiplicatif près, aux résultats obtenus pour les sols isotropes à partir de la formule de Boussinesq. C'est une propriété très importante, qui permet de généraliser aux milieux transversalement isotropes tous les résultats connus pour les sols isotropes. 

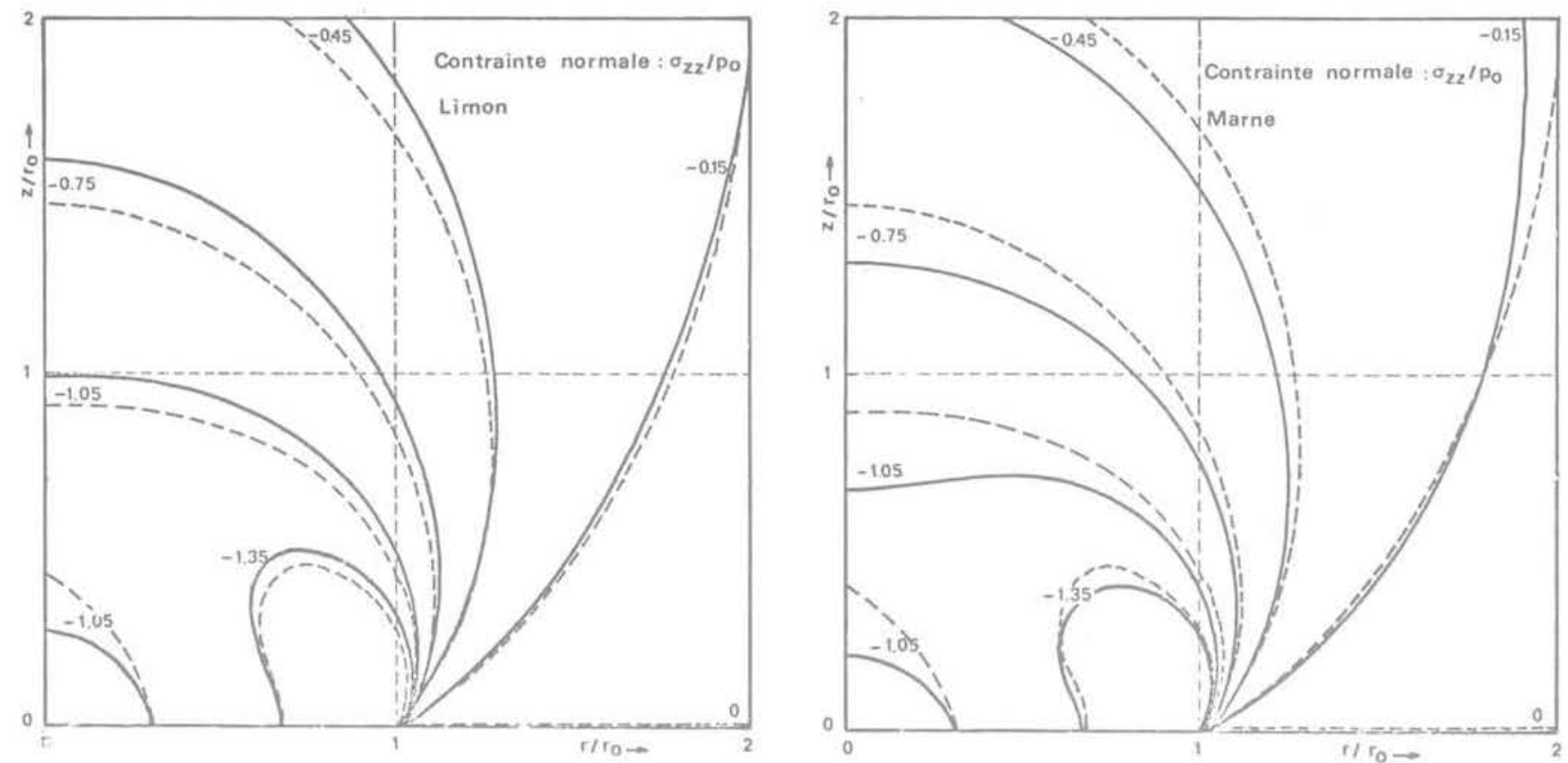

Fig. 1 Poinçon plat. Isovaleurs de la contrainte normale $\left(p_{0}=P / 2 \pi r_{0}^{2}\right)$
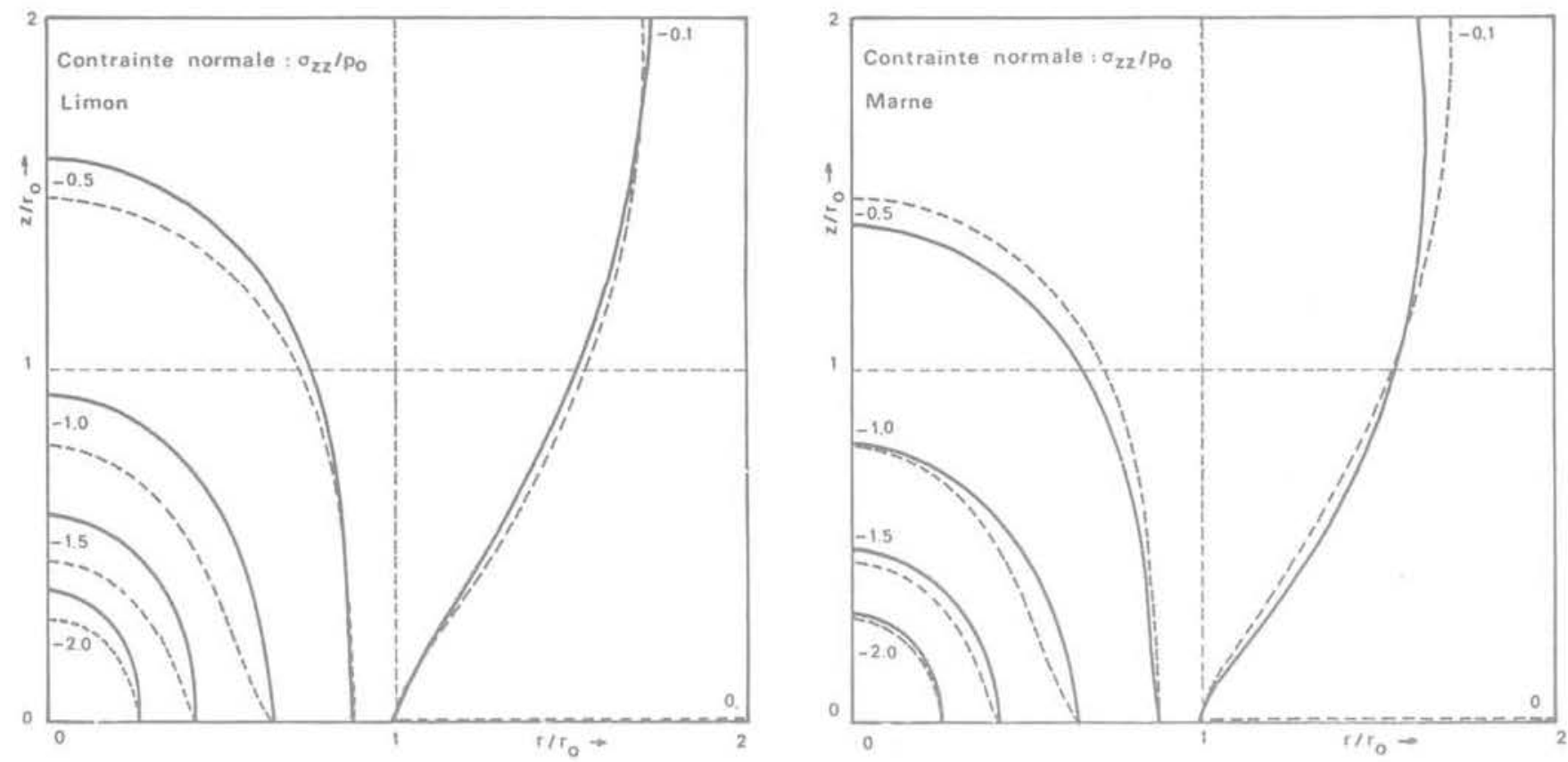

Fig. 2 Poinçon conique. Isovaleurs de la contrainte normale $\left(p_{0}=P / \pi r_{0}^{2}\right)$ 

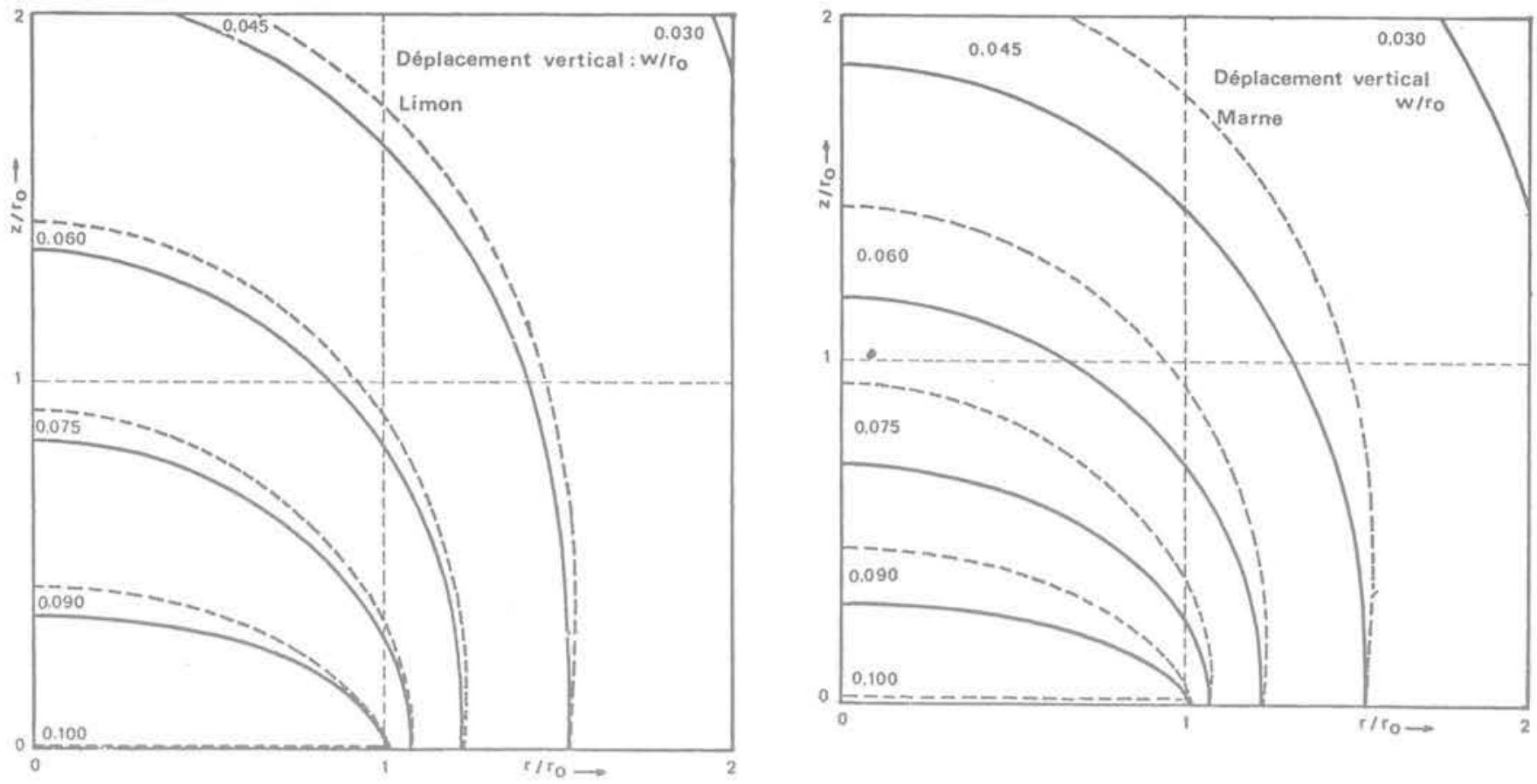

Fig. 3 Poinçon plat. Isovaleurs du déplacement vertical
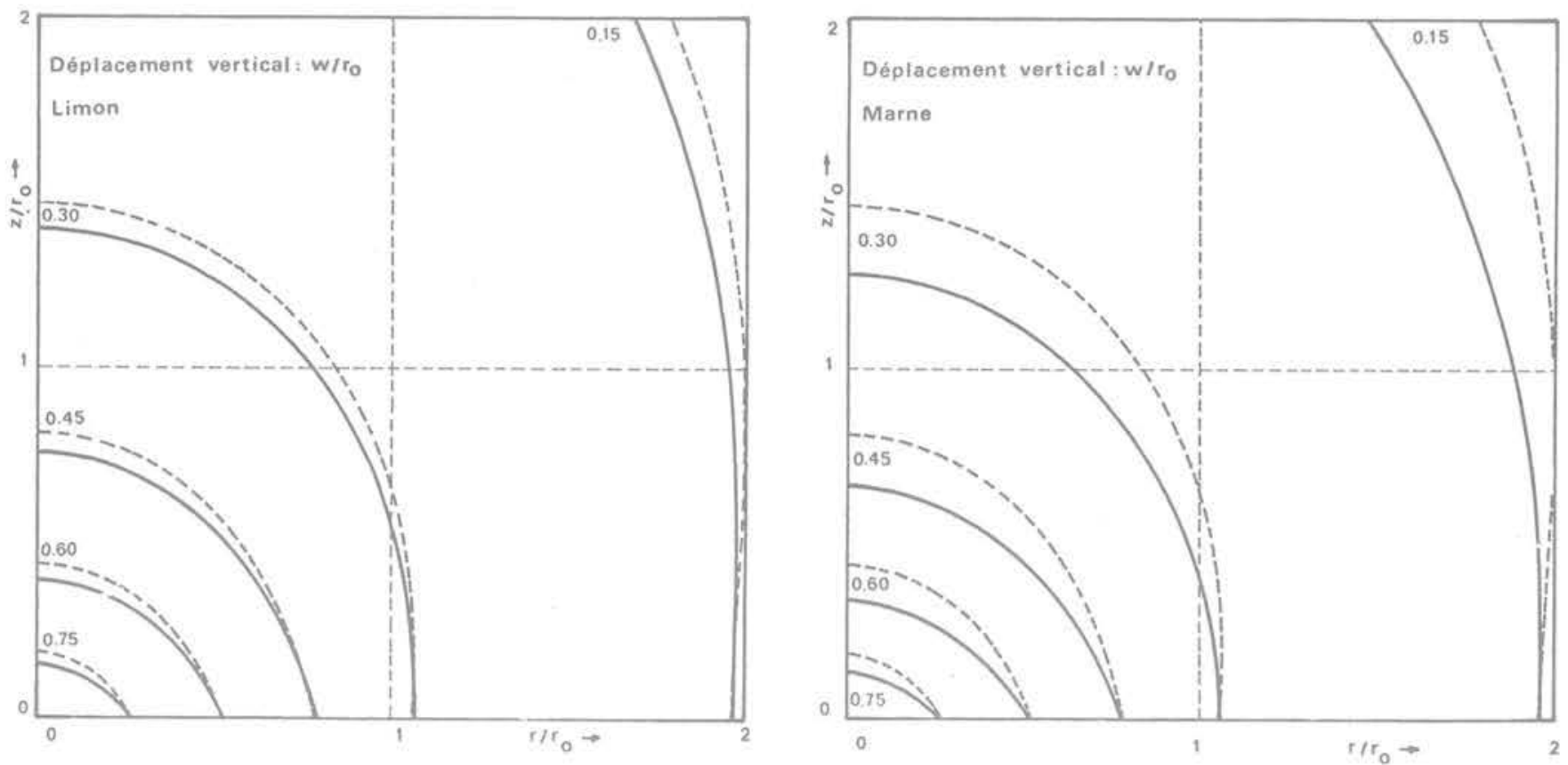

Fig. 4 Poincon conique. Isovaleurs de w

\section{Références bibliographiques}

BOUSSINESQ J. (1885) - Applications des potentiels à l'étude de l'équilibre ot du mouvement des solides élastiques. Gauthiers-Villars, Paris.

DAHAN M. (1975)- Poinçonnement élastique par une sphère d'un massif semi-infini transversalement isotrope. Thèse $3^{\circ}$ Cycle, Paris VI.

DAHAN M. (1979) - Poinçonnements axisymétriques rigides sur un massif semi-infini transversalement isotrope. A paraître, Journal de Mécanique Appliquée.

DAHAN M. et ZARKA J. (1977) - Elastic contact between a sphere and a semi-infinite transversely isotropic body. Int. J. Solids Structures, 13, 229-238.
DAHAN M., LUONG M.P. et MANDEL. J. (1978) - Sur I'anisotropie des sols. C.R. Acad. Sc. Paris, t. 287, B, 179-181. SNEDDON I.N. (1946) - Boussinesq's problems for a flatended cylinder. Proc. Camb. Phil. Soc., 42, 29-39. SNEDDON I.N. (1948) - Boussinesq's problem for a rigid cone. Proc. Camb. Phil. Soc., 44, 492-507.

SNEDDON I.N. (1965) - The relation between load and penetration in the axisymmetric Boussinesq's problem for a punch of arbitrary profile. Int. J. Eng. Sci., 3, 47-57. 